\title{
Lagrange Multiplier Based Domain Decomposition Method with Non-matching Grids for Time-dependent Equations
}

\author{
Wei Hong \\ People's Public Security University of China \\ Department of Science \\ Beijing, China \\ spt@midwest.com.cn
}

\author{
Sun Pengtao \\ Academia Sinica \\ The Institute of Mathematics \\ Beijing, China \\ spt@midwest.com.cn
}

\begin{abstract}
Our intention in this thesis is to study one kind of non-overlapping domain decomposed finite element method systematically - A Lagrange multiplier based domain decomposition method with non-matching grids for time-dependent problem. This method not only refers to methods defined on a decomposition of domain consisting of a collection of mutually disjoint subdomains, but also allows discontinuity of the interior variables across the boundary of the subdomains. We applied the method to parabolic equation, proposed its semi-discrete and fully discrete finite element approximated schemes of Lagrange multiplier based DDM with non-matching grids, studied their finite element solutions' existence and uniqueness, and obtained their optimal error estimate for $H^{1}$-norm and $L^{2}$-norm.
\end{abstract}

\section{Introduction}

We consider the following model problem

$$
\begin{cases}\frac{\partial u}{\partial t}-\Delta u=f, & (x, t) \in \Omega \times(0, T], \\ u=0, & (x, t) \in \partial \Omega \times(0, T], \\ u(x, 0)=u_{0}(x), & x \in \Omega,\end{cases}
$$

where $\Omega \subset R^{2}$ is a quasi-uniform polygonal domain with size d, $\partial \Omega$ is its boundary with piecewise Lipschitz smooth, $T>0$ is a constant, $f \in L^{2}\left(0, T ; L^{2}(\Omega)\right)$.

\section{Domain Decomposition and Finite El- ement Space}

We first decompose the domain $\Omega$ into subdomains $\Omega_{i}$, and then sub-divide every subdomain $\Omega_{i}$ into finite elements. We make the following assumption:

A1. $\Omega$ is a polygonal domain.

A2. $\Omega$ is decomposed into quasi-uniform subdomains $\Omega_{i}\left(i=1,2, \cdots, n_{d}\right)$ with size $d$. It means that there is a positive constant $c$ independent of $d$ such that every $\Omega_{i}$ contains a disk of diameter $c d$ and is contained in a disk of diameter $d$ and every side of $\Omega_{i}$ is greater than $c_{1} d, c_{1}$ independent of $d$.

A3. Every subdomain $\Omega_{i}$ is subdivided in the above sense into quasi-uniform triangular or quadrilateral elements with size $h_{i}$. Let $\Omega_{i}^{h_{i}}$ be the set of all elements in $\Omega_{i}, \Omega^{h}=\bigcup_{i} \Omega_{i}^{h_{i}}$.

Denote the vertices of $\Omega_{i}$ by $\gamma_{j}$ (in some order). $\Gamma_{i j}$ is the side of $\Omega_{i}$ with the vertices $\gamma_{i}$ and $\gamma_{j} . \Gamma=$ $\bigcup_{i=1}^{n_{d}} \partial \Omega_{i} / \partial \Omega$

We now construct the finite element space.

Let $S_{h_{i}}\left(\Omega_{i}\right)$ be the space of piecewise $m_{i}$-th degree continuous polynomials defined on $\Omega_{i}^{h_{i}}$. Define the space $S_{h}(\Omega)$, consisting of piecewise polynomials on $\Omega^{h}$,as follows:

$$
\begin{gathered}
S_{h}(\Omega)=\left\{f \mid f \text { is the piecewise } m_{i}\right. \text {-th degree } \\
\text { continuous polynomial on } \left.\Omega_{i}^{h_{i}}\right\} .
\end{gathered}
$$

Notice that the function in $S_{h}(\Omega)$ is only continuous in the interior of every subdomain but may be discontinuous on $\partial \Omega_{i}$. 
Let polynomial on $\left.\Gamma_{i j}\right\}$,

$$
S_{n}\left(\Gamma_{i j}\right)=\{f(x) \mid f(x) \text { is an n-th order }
$$

$\left.\partial \Omega_{i}\right\}$

$$
\begin{aligned}
& S_{n}(\Gamma)=\left\{\left.\lambda(\lambda)|\lambda|\right|_{\Gamma_{i j}} \in S_{n}\left(\Gamma_{i j}\right)\right\} \\
& S_{n}\left(\partial \Omega_{i}\right)=\left\{\lambda(\lambda)|\lambda|_{\Gamma_{i j}} \in S_{n}\left(\Gamma_{i j}\right), \Gamma_{i j} \subset\right.
\end{aligned}
$$

Notice that the function in $S_{n}(\Gamma)$ may be discontinuous at the vertices of the subdomains.

Define the finite element space by

$$
S_{h \times n}=S_{h}(\Omega) \times S_{n}(\Gamma),
$$

$(u, \lambda) \in S_{h \times n}$ if and only if $(u, \lambda) \in S_{h}(\Omega) \times S_{n}(\Gamma)$. Obviously, $S_{n}(\Gamma)=\left\{\lambda \mid(u, \lambda) \in S_{h \times n}\right\}$.

For the parameters $h_{i}, d, n$, we always assume that

$$
0<h_{i}<d, i=1,2, \cdots, n_{d},
$$

and

$$
\lim _{h \rightarrow 0} \frac{n^{2} h}{d}=0
$$

where $h=\max _{i}\left\{h_{i}\right\}$.

\section{Domain Decomposed Semi-discrete Finite element Method with non- matching Grids}

\subsection{Semi-discrete Finite Element Approxi- mated Scheme}

After we decompose $\Omega$ into a set of subdomain $\bigcup_{i=1}^{n_{d}} \Omega_{i}$, the weak form of $(1.1)$ is:

To find $(u, \lambda):[0, T] \rightarrow H(\Omega) \times H^{-\frac{1}{2}}(\Gamma)$,satisfying

$$
\left\{\begin{array}{l}
\sum_{i}\left\{\left(u_{t}, v\right)_{\Omega_{i}}+(\nabla u, \nabla v)_{\Omega_{i}}-\langle v, \lambda\rangle_{\partial \Omega_{i}}\right\} \\
=\sum_{i}(f, v)_{\Omega_{i}}, \\
\sum_{i}\langle u, \mu\rangle_{\partial \Omega_{i}}=0, \quad \forall(v, \mu) \in H(\Omega) \times H^{-\frac{1}{2}}(\Gamma),
\end{array}\right.
$$

where $H(\Omega)=H^{1}\left(\Omega_{1}\right) \oplus \ldots \oplus H^{1}\left(\Omega_{n_{d}}\right)$.

We now construct the semi-discrete finite element approximate scheme. To find $(U, \Lambda):[0, T] \rightarrow S_{h \times n}$, such that

$$
\left\{\begin{aligned}
\sum_{i}\left\{\left(U_{t}, v\right)_{\Omega_{i}}\right. & \left.+(\nabla U, \nabla v)_{\Omega_{i}}-\langle v, \Lambda\rangle_{\partial \Omega_{i}}\right\} \\
& =\sum_{i}(f, v)_{\Omega_{i}}, \\
\sum_{i}(U, \mu\rangle_{\partial \Omega_{i}}=0, & \\
U(x, 0)=\widetilde{u}_{0}(x), & \forall(v, \mu) \in S_{h \times n},
\end{aligned}\right.
$$

where $\widetilde{u}_{0}(x)$ is defined as following (3.3).

From [1] and Ladyzhenskaya-BabuškaBrezzi $(L B B)$ condition, it is easy to know finite element solution of problem (2) is existent and unique.

\subsection{Error Estimation}

To estimate the error between (3.2) and (3.1), we introduce a kind of projection about $\left(u, \frac{\partial u}{\partial n}\right)$, named $H^{1}$ projection, defined by finding $(\widetilde{u}, \widetilde{\lambda}):[0, T] \rightarrow S_{h \times n}$, such that:

$$
\left\{\begin{array}{cl}
\sum_{i}\left\{(\nabla(\widetilde{u}-u), \nabla v)_{\Omega_{i}}+(\widetilde{u}-u, v)_{\Omega_{i}}\right. & \\
\left.-\left\langle v, \widetilde{\lambda}-\frac{\partial u}{\partial n}\right\rangle_{\partial \Omega_{i}}\right\} & =0, \\
\sum_{i}\langle\widetilde{u}-u, \mu)_{\partial \Omega_{i}}=0, & \forall(v, \mu) \in S_{h \times n} .
\end{array}\right.
$$

It follows from $[1]$ that $(\widetilde{u}, \widetilde{\lambda})$ is existent and unique. The error estimation between $(\tilde{u}, \tilde{\lambda})$ and $\left(u, \frac{\partial u}{\partial n}\right)$ satisfy the lemma as follow:

We analyzed the error estimation between (3.2) and (3.1), and obtain the following theorem

Theorem 1 Let $h<d, m \leq n, k \geq 2 m, u$ is the mathematical solution of problem (1.1), and $u \in$ $W^{1,2}\left(0, T ; H^{k+1}\left(\Omega_{i}\right)\right), i=1, \ldots, n_{d},(U, \Lambda)$ is the finite element solution of (2), then

$$
\begin{aligned}
& \left(\sum _ { i } \left(\|U-u\|_{L^{\infty}\left(L^{2}\left(\Omega_{i}\right)\right)}^{2}+\left\|(U-u)_{t}\right\|_{L^{2}\left(L^{2}\left(\Omega_{i}\right)\right)}^{2}\right.\right. \\
& \left.\left.+\left(h+n^{-1} d^{\frac{1}{2}}\right)^{2}\|U-u\|_{L^{\infty}\left(H^{1}\left(\Omega_{i}\right)\right)}^{2}\right)\right)^{\frac{1}{2}} \\
& +\left(h+n^{-1} d^{\frac{1}{2}}\right)^{2}\left\|\Lambda-\frac{\partial u}{\partial n}\right\|_{L^{2}\left(H^{-\frac{1}{2}}\left(\partial \Omega_{i}\right)\right)}^{2}{ }^{\infty}\left(h+n^{-1} d^{\frac{1}{2}}\right) \cdot\left(\sum _ { i } \left(\|u\|_{L^{2}\left(H^{m+1}\left(\Omega_{i}\right)\right)}^{2}\right.\right. \\
& +\left\|u_{t}\right\|_{L^{2}\left(H^{m+1}\left(\Omega_{i}\right)\right)}^{2}+\|u\|_{L^{2}\left(H^{k+\frac{1}{2}}\left(\partial \Omega_{i}\right)\right)}^{2} \\
& \left.\left.+\left\|u_{t}\right\|_{L^{2}\left(H^{\left.k+\frac{1}{2}\left(\partial \Omega_{i}\right)\right)}\right.}^{2}\right)\right)^{\frac{1}{2}} .
\end{aligned}
$$

4. Domain Decomposed Full-discrete Finite element Method with nonmatching Grids

\subsection{Full discrete Finite Element Approxi- mated Scheme}

First of all,we decomposed equally the time domain $[0, T]$ into $N$ parts: $0=t_{0}<t_{1}<\ldots<t_{N}=T, \tau=$ $t_{j+1}-t_{j}=\frac{T}{N}, t_{j}=j \tau$. We introduce some notations

$$
v^{j}=v\left(x, t_{j}\right), j=0,1, \cdots, N
$$




$$
\begin{gathered}
v^{j+\frac{1}{2}}=\frac{1}{2}\left(v^{j+1}+v^{j}\right), \partial_{t} v^{j+\frac{1}{2}}=\frac{1}{\tau}\left(v^{j+1}-v^{j}\right), \\
j=0,1, \cdots, N-1 .
\end{gathered}
$$

The full-discrete approximation for domain decomposition method with non-matching grids is defined by the sequence, find $\left(\left\{U^{j}\right\}_{j=0}^{N},\left\{\Lambda^{j}\right\}_{j=0}^{N}\right):[0, T] \rightarrow$ $S_{h \times n}$, such that

$$
\left\{\begin{array}{l}
\sum_{i}\left\{\left(\partial_{t} U^{j+\frac{1}{2}}, v\right)_{\Omega_{i}}+\left(\nabla U^{j+\frac{1}{2}}, \nabla v\right)_{\Omega_{i}}\right. \\
\left.-\left\langle v, \Lambda^{j+\frac{1}{2}}\right\rangle_{\partial \Omega_{i}}\right\} \\
\quad=\sum_{i}\left(f^{j+\frac{1}{2}}, v\right)_{\Omega_{i}}, \\
\sum_{i}\left\langle U^{j+1}, \mu\right\rangle_{\partial \Omega_{i}}=0 \\
U^{0}=\widetilde{u}_{0}(x), \forall(v, \mu) \in S_{h \times n}, j=0,1, \ldots, N-1,
\end{array}\right.
$$

where $\widetilde{u}_{0}(x)$ is defined by $(3.3)$.

\subsection{Algorithm's description}

1.Guess $U^{0}$ initially, the $\mathrm{j}$ step of time $=0, \mathrm{t}=0$.

2. Given $\mathrm{j}=\mathrm{j}+1, t=t+\tau$ until $j=N, t=T$, we can compute respective stiffness and vector on each time step: $M_{i}, S_{i}, L_{i}$ and $F_{i}$, which is local boundary stiffness matrix $L_{i}^{T^{\prime}}\left(M_{i}+\frac{\tau}{2} S_{i}\right)^{-1} L_{i}$ and local equivalent load $-L_{i}^{T}\left(M_{i}+\frac{r}{2} S_{i}\right)^{-1}\left(\frac{\tau}{2}\right)^{-1} F_{i}$. The total boundary stiffness matrix $S$ and the total equivalent load $F$ can be obtained by summation over them.

3. Solve $S \Lambda^{j+1}=F$ by iteration, where $\Lambda^{j+1}$ is the unknown on the boundary, consisting of $\Lambda_{i}^{j+1},(i=$ $\left.1, \cdots, n_{d}\right)$.

4.Find $U^{j+1}$ in (4.4), $\left(i=1, \ldots, n_{d}\right)$.

5.Go to the second step and continue the time step's iteration.

In implementation, we only need the local boundary stiffness matrix and the local equivalent load since we solve $S \Lambda^{j+1}=F$ by iteration. The algorithm is suitable to parallel computing.

\subsection{Error Estimation}

We list out the theorem of error estimate between (4.1) and (3.1), where we use the $H^{1}$ projection $(\widetilde{u}, \widetilde{\lambda})$ which was based on Lagrange multiplier of DDM and has been defined in $\S 2$. Notations $\xi, \eta, \zeta, \theta$ are the same as that in $\S 2$.

Theorem 2 If $u_{t^{3}} \in L^{2}\left(0, T ; L^{2}\left(\Omega_{i}\right)\right), i=1, \cdots, n_{d}$, $\left(\left\{U^{j}\right\}_{j=0}^{N},\left\{\Lambda^{j}\right\}_{j=0}^{N}\right)$ is the finite element solution of
(4.1), then under the conditions of Theorem1, we have

$$
\begin{aligned}
& \sup _{1 \leq M \leq N}\left\{\left\|U^{M}-u^{M}\right\|_{0, \Omega}\right. \\
& +\left(\tau \sum_{j=0}^{M-1}\left\|\partial_{t}\left(U^{j+\frac{1}{2}}-u^{j+\frac{1}{2}}\right)\right\|_{0, \Omega}^{2}\right)^{\frac{1}{2}} \\
& +\left(h+n^{-1} d^{\frac{1}{2}}\right)\left(\sum_{i}\left\|U^{M}-u^{M}\right\|_{1, \Omega_{i}}^{2}\right)^{\frac{1}{2}} \\
& \left.+\left(h+n^{-1} d^{\frac{1}{2}}\right)\left(\tau \sum_{j=0}^{M-1} \sum_{i}\left|\Lambda^{j+\frac{1}{2}}-\left(\frac{\partial u}{\partial n}\right)^{j+\frac{1}{2}}\right|_{-\frac{1}{2}, \partial \Omega_{i}}^{2}\right)^{\frac{1}{2}}\right\} \\
& \lesssim\left\{h^{m}\left(h+n^{-1} d^{\frac{1}{2}}\right)+\tau^{2}\right\}\left\{\left(\sum_{i}\|u\|_{L^{2}\left(\boldsymbol{H}^{m+1}\left(\Omega_{i}\right)\right)}^{2}\right)^{\frac{1}{2}}\right. \\
& +\left(\sum_{i}\|u\|_{L^{2}\left(H^{k+\frac{1}{2}}\left(\partial \Omega_{i}\right)\right)}^{2}\right)^{\frac{1}{2}}+\left(\sum_{i}\left\|u_{t}\right\|_{L^{2}\left(H^{m+1}\left(\Omega_{i}\right)\right)}^{2}\right)^{\frac{1}{2}} \\
& \left.+\left(\sum_{i}\left\|u_{t}\right\|_{L^{2}\left(H^{\left.k+\frac{1}{2}\left(\partial \Omega_{i}\right)\right)}\right.}^{2}\right)^{\frac{1}{2}}+\left(\sum_{i}\left\|u_{t^{3}}\right\|_{L^{2}\left(L^{2}\left(\Omega_{i}\right)\right)}^{2}\right)^{\frac{1}{2}}\right\} .
\end{aligned}
$$

\section{References}

[1] Guoping Liang, Jiangheng He,Incompatible Domain Decompositon Lagrangian Multiplier. J. Comp. Math. , No.2(1992), pp207-215.

[2] Howard Swann, On the use of Lagrange multipliers in domain decomposition for solving elliptic problems. Math. Comp., 60(1993), pp49-78.

[3] C.Farhat, J.Mandel, \& F.X.Roux, Optimal convergence properties of the FETI domain decomposition method. Comput. Methods Appl. Mech. Eng., 115(1994), pp367-388.

[4] J.Mandel, R.Tezaur, Convergence of a substructuring method with Lagrange multipliers. $U C D / C C M$ Report 33, University of Colorado at Denver,1994.

[5] P.Le,Tallec,T.Sassi, \& M.Vidrascu, Threedimensional domain decomposition methods with nonmatching grids and unstructured coarse solvers. Contemporary Mathematics, 180(1994), pp61-74.

[6] C.Farhat, J.Mandel, \& Po-shu Chen, A scalable Lagrange multiplier based domain decomposition method for time-dependent problems. Int. J. Numer. Methods Eng., 38(1995), pp3831-3853.

[7] C.Farhat, F.X.Roux, Implicit parallel processing in structural mechanics. Comp. Mech. Adv., 2(1994), pp1-124.

[8] Guoping Liang, Ping Liang, Domain Decomposition Method of Hybrid Finite Element, J. Comp. Math. ,No.3(1989), pp363-370. 